\title{
On Andrica's Conjecture, Cramér's Conjecture, gaps Between Primes and Jacobi Theta Functions II: A Simple Proof of Asymptotic for Andrica's Conjecture
}

\section{Prof. Dr. Raja Rama Gandhi and Edigles Guedes}

${ }^{1}$ Resource person in Math for Oxford University Press, Professor in Math, BITS-Vizag.

${ }^{2}$ World order Number Theorist, Pernambuco, Brazil.

\section{PRELIMINARES}

In [1, p. 185] states that the prime number theorem yields:

$$
p_{n} \sim n \log n,
$$

that is,

LEMMA 1. The Andrica's conjecture is equivalent to

$$
\lim _{n \rightarrow \infty} \frac{p_{n}}{n \log n}=1
$$

Proof. Step 1. In [2, p.__ ], we conclude that Andrica's onjecture is equivalent to

$$
p_{n+1}<1+2 \sqrt{p_{n}} p_{n}
$$

$$
\sqrt{p_{n}}<\frac{\theta_{2}}{\theta_{3}-c} \Leftrightarrow \frac{1}{\theta_{2}}<\frac{1}{\sqrt{p_{n}}}
$$

where $k:=\frac{p_{n}}{p_{n+1}}$ is a $k$ modulus. In [3 - 3 ], we c counter

2. THEOREM

THEOREM 1 (Asymptotic for Andrica's Conjecture). Let $n \in \mathbb{N}$ and $n$ sufficiently large, then

$$
\sqrt{p_{n+1}}-\sqrt{p_{n}}<1
$$

Proof. Henceforth, we will use the reductio ad absurdum to prove the Lemma 1. We assume that

$$
p_{n+1} \geq 1+2 \sqrt{p_{n}}+p_{n}
$$


For $n$ sufficiently large, we set (1) in (7), as follows

$$
(n+1) \log (n+1) \geq 1+2 \sqrt{n \log n}+n \log n .
$$

Dividing ( 8 ) by $\log (n+1)$, we encounter

$$
n+1 \geq \frac{1}{\log (n+1)}+2 \frac{\sqrt{n \log n}}{\log (n+1)}+n \frac{\log n}{\log (n+1)} .
$$

On the other hand, it is easy to see that, as $n$ is sufficiently large, then

$$
\frac{\log n}{\log (n+1)} \rightarrow 1, \quad \frac{1}{\log (n+1)} \rightarrow 0, \quad \frac{\sqrt{n \log n}}{\log (n+1)} \rightarrow \infty
$$

namely,

$$
\lim _{n \rightarrow \infty} \frac{\log n}{\log (n+1)}=1, \quad \lim _{n \rightarrow \infty} \frac{1}{\log (n+1)}=0,
$$

Substituting (10) in (9), we find

$$
n+1 \geq 0+2(\infty)+n \cdot 1 \Leftrightarrow 2(\infty)
$$

which is false. Therefore, for $n$ sufficiently large, $p_{n+1}<1+2 \sqrt{p_{n}}+p_{n}$. In face of Lemma 1 , the asymptotic for Andrica's conjecture is proved.

\section{ACKNOWLEDGMENTS}

I thank Prof. Dr. K. Raja Rama Gandi ad yyar society for their encouragement and support during the development of thi

\section{REFERENCES}

[1] Ribenboim, Paulo, The tle Book o, ioger, Springer, 2000.

[2] Prof. Dr. Raja Ran Gan and Guedes, Edigles, On Andrica's Conjecture, gaps Between Primes and Jacobj heta Function

[3] Armitage, 万. nn' Eberle n, W. F., Elliptic Functions, London Mathematical Society, 2006.

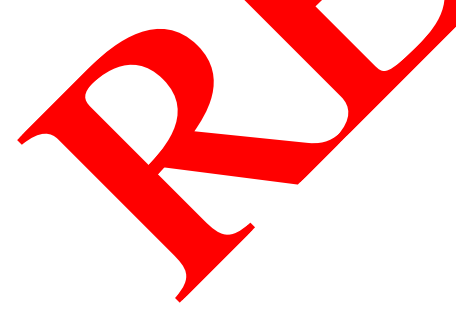

